\title{
Significance of Outer Keratin Cortices to Structural Color Production in Feathers Revealed through Focused Ion Beam Milling and Microspectrometry.
}

\author{
Matthew D. Shawkey*1, Nanthawan Avishai**, Amir Avishai** \\ * Department of Biology and Integrated Bioscience Program, University of Akron, Akron, OH \\ 44325,1shawkey@uakron.edu \\ ** Department of Material Science and Engineering, Case Western Reserve University, \\ Cleveland, $\mathrm{OH} 44106$
}

Coloration of metazoans is produced by differential absorption of light by pigments (e.g. carotenoids, melanins) and/or by scattering of light from biological nanostructures (i.e. referred to as "structural color") [1,2]. These nanostructures have diverse morphologies ranging from laminar arrays to three-dimensional matrices and produce a startling array of colors from deep ultraviolet to red [3]. In birds, non-iridescent structural feather colors are typically created by matrices of keratin and air forming a single medullary layer within feather barbs (termed a "spongy layer"), while iridescent colors are typically created by stacks of melanin granules within a keratin substrate in feather barbules [3]. All of these nanostructures are bounded by an unstructured layer of keratin that varies widely in uniformity and thickness between species. While this cortex is known to play a critical role in color production of $1 \mathrm{D}$ thin film nanostructures, its role (if any) in other nanostructures is unknown.

We therefore used focused ion beam (FIB) milling (without prior coating) to experimentally remove portions of this cortex from barbules of a wild turkey (Mellagris gallopavo) feather with a 2D hexagonal nanostructure and barbs of a blue dacnis (Dacnis cyana) feather with a 3D spongy layer nanostructure. FIB milling was carried out using a $30 \mathrm{keV}$ ion beam and a current of $100 \mathrm{pA}$, on a dual beam FIB, Nova Nano-lab 200, FEI Company. As a control, we removed cortex of barbules from rock pigeon (Columba livia) feathers with a 1D nanostructure in which thickness of the cortex is known to determine reflected color. To identify effects of cortex removal, we used microspectrometry to compare color of milled sections with adjacent nonmilled sections. When the cortex was entirely removed, both the rock pigeon and wild turkey barbules lost all color, while color of the dacnis feather did not change. Moreover, when cortex was partially removed, color of both the pigeon and turkey feather shifted to lower wavelength hues, indicating that the cortex contributes to color production. Partial removal of the dacnis cortex was unsuccessful, but the fact that total removal did not change its color suggests that the cortex is not as important in this type of nanostructure.

While methodological details such as ion beam voltage and current need to be resolved further, this technique provides a promising experimental approach to address significant questions in the study of structural color.

[1] D. L. Fox. Animal biochromes and structural colours. Berkeley, CA: University of California Press (1976). 
[2] G. E. Hill and K. J. McGraw (eds). 2006. Bird Coloration vol. I: Mechanisms and measurements. Boston: Harvard University Press.

[3] M. D. Shawkey et al., J. Roy. Soc. Interface. 6 (2009) S221.
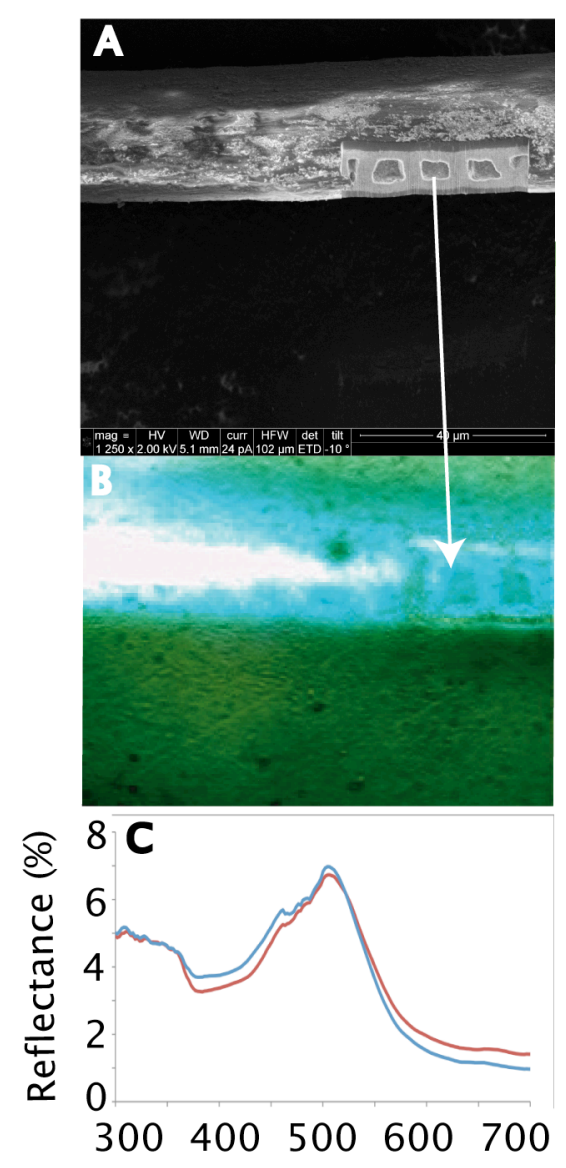
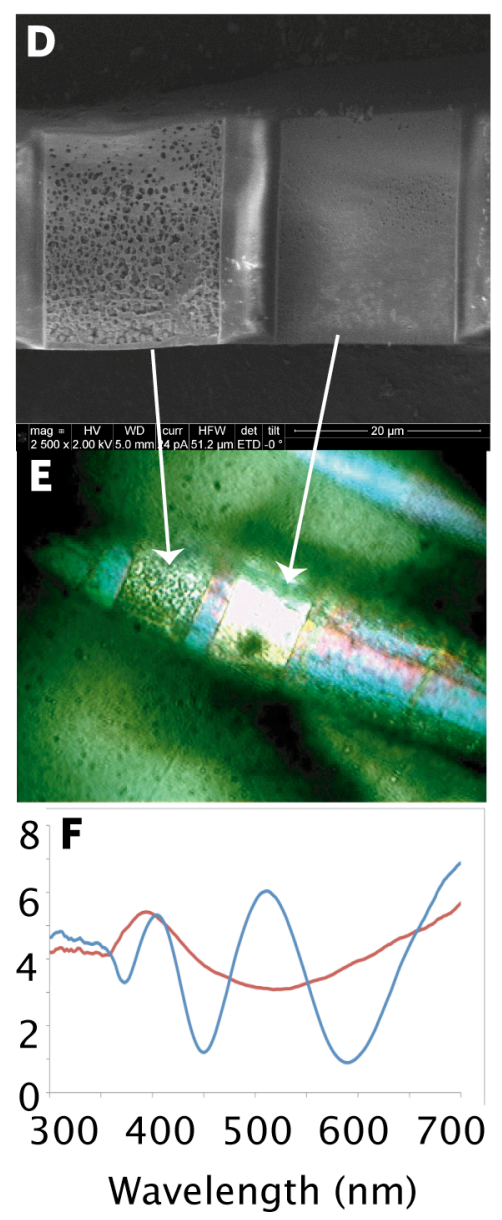
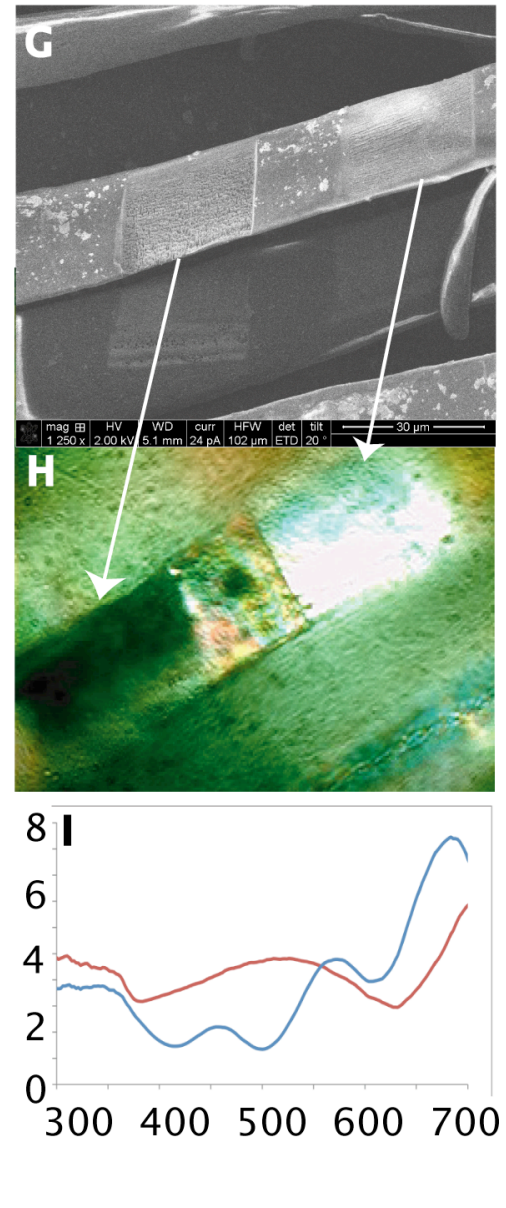

Figure 1: Effects of cortex removal through FIB milling on structural color of barbs of blue dacnis (A-C), and barbules of rock pigeon (D-F) and wild turkey (G-I) feathers. A,D,G: SEM images of barbs/barbules showing milled and unmilled areas of the cortex. Arrows from milled areas point to the same areas seen in reflected light microscope images (B,E,H). UV-vis spectrometry curves were measured from milled and unmilled areas (C,F,I). Blue curves indicate values for unmilled areas while red curves indicate values for milled areas. 